\title{
Patologia da carótida
}

Inês Müller Pinheiro,* Gil Marques, ${ }^{* *}$ Nádia Duarte, ${ }^{*}$ Ana Gonçalves, ${ }^{* *}$ Pedro Barroso, ${ }^{* *}$ António Gonzalez, ${ }^{* *}$ Maria José Ferreira***

\section{RESUMO}

A aterosclerose é responsável por cerca de $90 \%$ da doença cerebrovascular extracraniana. As placas ateroscleróticas ocorrem, preferencialmente, na bifurcação carotídea e a sua consequência mais temida é o acidente vascular cerebral (AVC), que constitui a primeira causa de morbilidade e incapacidade permanente na Europa.

Considera-se, além disso, que é a segunda causa mais importante de demência, a causa mais importante de epilepsia nos idosos e uma causa frequente de depressão.

Aproximadamente $80 \%$ dos AVC são isquémicos e dois terços resultam de doença aterosclerótica carotídea extracraniana, consequência de embolização de uma placa na bifurcação carotídea para os vasos intracranianos ou por baixo débito. 0 ecodoppler carotídeo está recomendado como exame de primeira linha no diagnóstico. Os objectivos do tratamento médico são a prevenção da oclusão ou da embolização arterial.

As guidelines actuais para a prevenção do AVC incluem recomendações para alterações do estilo de vida e o controlo dos factores de risco. Na prevenção de eventos tromboembólicos em doentes com aterosclerose carotídea, os fármacos indicados são os antiagregantes plaquetários. O tratamento cirúrgico (endarterectomia ou stenting) está indicado em todos os doentes que apresentem uma estenose carotídea sintomática superior a 70\%. Uma estenose carotídea assintomática, de 60 a $99 \%$, apenas terá indicação para endarterectomia em doentes com alto risco para AVC, não estando demonstrada a indicação do stent carotídeo neste grupo.

O Médico de Família deverá identificar os grupos de risco para esta patologia, promover a correcção dos factores de risco, fazer o diagnóstico precoce e instituir o tratamento médico recomendado, tendo conhecimento das indicações cirúrgicas para uma correcta referenciação a Cirurgia Vascular.

Palavras-chave: Acidente Cerebral Vascular; Aterosclerose; Doença Carotídea; Inibidores da Agregação de Plaquetas.

\section{EPIDEMIOLOGIA E MANIFESTAÇÕES CLÍNICAS}

$\mathrm{A}$ aterosclerose é responsável por cerca de $90 \%$ da doença cerebrovascular extracraniana, ocorrendo placas ateroscleróticas preferencialmente na bifurcação carotídea. A sua consequência mais temida é o acidente vascular cerebral (AVC), que é a primeira causa de morbilidade e incapacidade permanente na Europa. ${ }^{1}$

Considera-se, ainda, como a segunda causa mais importante de demência, a causa mais importante de epilepsia nos idosos e uma causa frequente de depressão. ${ }^{1}$

Aproximadamente $80 \%$ dos AVC são isquémicos e dois terços resultam de doença aterosclerótica carotídea extracraniana, consequência da embolização de

*Interna do Internato Complementar de Angiologia e Cirurgia Vascular, Serviço de Angiologia e Cirurgia Vascular do Hospital Garcia de Orta.

**Assistente Hospitalar de Angiologia e Cirurgia Vascular, Serviço de Angiologia e Cirurgia Vascular do Hospital Garcia de Orta.

***Directora do Serviço de Angiologia e Cirurgia Vascular do Hospital Garcia de Orta uma placa na bifurcação carotídea para os vasos intracranianos ou por baixo débito. ${ }^{2,3,4,5}$

A idade avançada, o sexo masculino e a raça são factores de risco para AVC e para a doença carotídea aterosclerótica. Existe maior risco de AVC cardioembólico na raça caucasiana, sendo o AVC aterosclerótico mais frequente nas raças negra e hispânica. Os factores de risco cardiovascular habituais, como a hipertensão, diabetes, tabagismo e doença cerebrovascular conhecida são os outros factores importantes a considerar. ${ }^{2,3} \mathrm{O}$ tabagismo é considerado um factor de risco independente para AVC isquémico em ambos os sexos. ${ }^{6}$ Apesar da hipercolesterolémia ter sido associada a aumento do risco para doença coronária, enfarte agudo do miocárdio e outros eventos cardiovasculares, a sua associação com o AVC tem sido inconsistente. ${ }^{3} \mathrm{O}$ consumo de álcool superior a $60 \mathrm{gr} /$ dia aumenta o risco de AVC isquémico (RR 1,69) e hemorrágico (RR 2,18)

Os doentes com doença carotídea podem ser classi- 
ficados em assintomáticos ou sintomáticos. Os sintomas relacionados com lesões da artéria carótida são geralmente classificados como AVC ou Acidente Isquémico Transitório (AIT) (Quadro 1), sendo estes últimos definidos como défices neurológicos de início súbito, que duram menos de $24 \mathrm{~h}$. Os AIT representam um factor de risco major para AVC e todos devem ser avaliados e tratados agressivamente. ${ }^{2}$

\section{DIAGNÓSTICO}

O diagnóstico da doença da bifurcação carotídea é facilitado pela localização relativamente superficial da artéria carotídea, que a torna acessível à auscultação e à palpação. $\mathrm{O}$ pulso carotídeo cervical é geralmente normal em doentes com doença da bifurcação carotídea, visto a artéria carótida comum ser o único vaso palpável no pescoço e raramente se encontrar significativamente doente. Sopros carotídeos podem ser auscultados anteriormente ao músculo esternocleidomastoideu, perto do ângulo da mandíbula. Só se tornam audíveis quando a estenose é suficientemente severa para reduzir o diâmetro do lúmen em, pelo menos, $50 \% .4,5$

Em 2006, o U.K. Health Technology Assessment publicou uma meta-análise ${ }^{4}$ de estudos que avaliavam os meios de imagem não invasivos no diagnóstico desta patologia e concluiu que, apesar da angio-ressonância

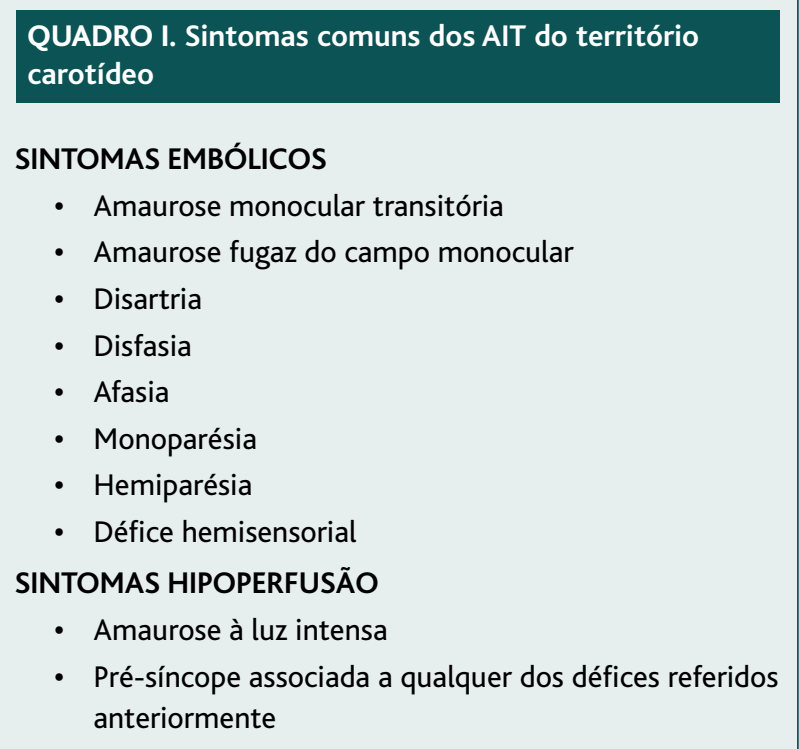

AIT - acidente isquémico transitório. magnética nuclear (angio-RMN) ser o melhor método de imagem, a sua eficácia clínica encontrava-se limitada pela inacessibilidade, falta de disponibilidade e atrasos na sua realização. Tendo em conta estes aspectos, concluiu que o ecodoppler carotídeo permanecia o método de imagem preferido para identificar doentes com estenose na artéria carótida interna, devido ao seu baixo custo, maior número de AVC que poderiam ser prevenidos a longo prazo, a sua robustez nas análises de sensibilidade e a sua capacidade de corresponder às necessidades da cirurgia (em termos de localização da bifurcação, tipo de placa e quantificação do grau de estenose). A determinação da extensão da estenose no ecodoppler carotídeo é baseada no critério da velocidade sistólica nos vasos carotídeos.

$\mathrm{O}$ grau de estenose deve ser classificado de acordo com os critérios da North American Symptomatic Carotid Endarterectomy Trial (NASCET). ${ }^{4,5}$

Um exame de imagem suplementar, como a angioRMN, será necessário sempre que houver incertezas acerca dos achados no ecodoppler, estiver planeada uma intervenção em casos com benefício borderline face à gravidade da estenose (doentes sintomáticos com estenose de 50 a $69 \%$ ) ou em qualquer doente que vá ser submetido a stenting carotídeo. ${ }^{4,5}$

Para além dos exames de imagem, todos os doentes com doença carotídea deverão fazer exames laboratoriais no sentido de se determinar o risco cirúrgico ou detectar factores de risco. Um hemograma completo deve excluir trombocitopénia (que implica risco potencial de hemorragia e cuidados com a escolha e dose do agente antiagregante), trombocitose (que poderá indicar uma tendência protrombótica), anemia (possível neoplasia) ou policitémia (tendência protrombótica). $\mathrm{O}$ estudo bioquímico de base deve identificar disfunção renal (importante no caso de se planear stenting carotídeo), hiperlipidémia e diabetes não diagnosticada. ${ }^{3}$

Em casos específicos, deve ser investigada a possibilidade de uma patologia não ateromatosa ou protrombótica ser a responsável pela doença carotídea. Nos doentes com menos de 50 anos, que tenham tido um AVC/AIT ou tenham uma história pessoal/familiar de trombose deve ser feito o estudo das trombofilias (Quadro II) e ser obtido o nível da homocisteína em jejum. ${ }^{3}$

No futuro, poderá haver um papel para a medição de biomarcadores plasmáticos (como, por exemplo, a pro- 
teína $C$ reactiva de elevada sensibilidade e metaloproteinase 9 da matriz), especialmente em doentes com doença carotídea assintomática, no sentido de seleccionar os doentes que se encontram em risco elevado de AVC. ${ }^{3}$ Testar a resistência à aspirina ou clopidogrel poderá, também, no futuro, permitir uma melhor selecção da terapêutica antiagregante mais apropriada para cada doente. ${ }^{3}$

\section{RASTREIO E SEGUIMENTO}

Em 2007, a U.S. Preventive Service Task Force recomendou contra o rastreio de todos os doentes com idade superior a 55 anos visto este procedimento não ser custo-efectivo. ${ }^{5}$ No entanto, o ecodoppler carotídeo está recomendado como exame de primeira linha em coortes seleccionadas, como doentes com doença arterial periférica ou doença coronária (presença de doença aterosclerótica noutros territórios), embora não existam indicações sobre a sua periodicidade. ${ }^{5}$

A vigilância com ecodoppler anual poderá ser benéfica em doentes nos quais tenha sido detectada estenose carotídea ligeira a moderada (inferior a 50\%) e

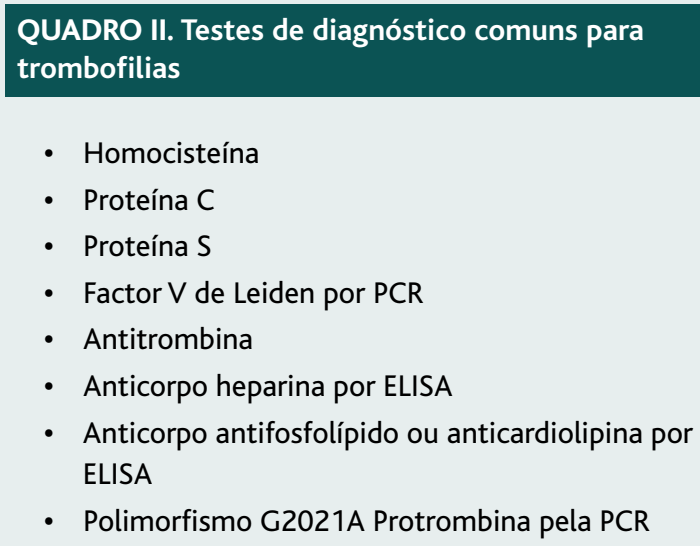

\section{QUADRO III. Terapêutica médica}

\begin{tabular}{|c|c|c|}
\hline $\begin{array}{l}\text { Alterações de estilos } \\
\text { de vida }\end{array}$ & $\begin{array}{l}\text { Controlo dos } \\
\text { factores de risco }\end{array}$ & $\begin{array}{l}\text { Antiagregantes } \\
\text { plaquetários }\end{array}$ \\
\hline $\begin{array}{l}\text { - Perda de peso } \\
\text { - Exercício físico regular } \\
\text { - Dieta saudável } \\
\text { - Abandono do tabagismo }\end{array}$ & $\begin{array}{l}\text { - Controlo da tensão arterial } \\
\text { - Controlo da glicémia } \\
\text { - Controlo da hiperlipidémia } \\
\text { com estatinas }\end{array}$ & $\begin{array}{l}\text { - Aspirina } 150 \text { mg/dia } \\
\text { - Clopidogrel } 75 \text { mg/dia }\end{array}$ \\
\hline
\end{tabular}

deve ser efectuada em todos os doentes sujeitos a intervenção cirúrgica para se detectar reestenose. ${ }^{5}$ No nosso serviço, o seguimento dos doentes, após a intervenção, é feito no nosso laboratório vascular aos seis meses, um ano e, depois, anualmente.

\section{TERAPÊUTICA}

O objectivo principal do tratamento da doença carotídea é a prevenção do AVC, que permanece a principal causa de morte em Portugal. Cerca de 15\% dos AVC são fatais e $15-20 \%$ resultam numa incapacidade funcional importante; cerca de $15-20 \%$ dos doentes que recuperam irão sofrer um AVC subsequente no futuro. ${ }^{2,3}$

\section{Terapêutica médica}

Os objectivos do tratamento médico são a prevenção da oclusão arterial e da embolização arterial. A intervenção médica deve complementar a intervenção cirúrgica para tratar a doença carotídea e incluir medidas de controlo dos factores de risco para aterosclerose acelerada ou terapêutica antitrombótica.

As guidelines actuais para a prevenção do AVC incluem recomendações para alteração dos estilos de vida como perda de peso, exercício físico regular, consumo limitado de álcool e de gorduras e abandono do tabagismo. ${ }^{8}$

A alteração dos estilos de vida deve ser complementada pelo controlo dos factores de risco do doente. Existe evidência na literatura de que o controlo da tensão arterial reduz o risco de AVC. ${ }^{8}$ Os inibidores da enzima de conversão da angiotensina (IECA) e os antagonistas dos receptores da angiotensina II (ARA II) diminuem a tensão arterial e poderão diminuir a progressão da doença aterosclerótica. ${ }^{6} \mathrm{~A}$ hiperlipidémia causa uma progressão da aterosclerose cerebrovascular. As estatinas diminuem a progressão da doença carotídea, estabilizam a parede arterial e, no período perioperatório, poderão, também, diminuir os eventos adversos associados, quer à endarterectomia carotídea, quer ao stenting carotídeo. ${ }^{6} \mathrm{O}$ período de tempo mínimo, prévio à cirurgia, para o qual se encontram estes benefícios não está definido, embora alguns estudos pareçam mostrar que uma estati- 
na iniciada uma semana antes tenha efeitos benéficos. Não existe nenhuma recomendação para preferir uma estatina sobre outra, podendo ser todas usadas, nas dosagens habitualmente escolhidas na terapêutica da dislipidémia associada a outras patologias. Pretendem-se valores alvo de $\mathrm{LDL}<100 \mathrm{mg} / \mathrm{dL}$ e de $\mathrm{HDL}>50 \mathrm{mg} / \mathrm{dL} ;{ }^{6}$ caso se trate de um diabético, os valores de LDL devem ser inferiores a $70 \mathrm{mg} / \mathrm{dL} .{ }^{6}$ São, igualmente, recomendadas modificações na dieta e terapêutica farmacológica, para que os diabéticos mantenham uma hemoglobina glicosilada (HbAlc) inferior a $7 \%{ }^{8}$

Na prevenção de eventos tromboembólicos em doentes com aterosclerose carotídea os fármacos indicados são os antiagregantes plaquetários. São eficazes na prevenção do AVC isquémico, enfarte agudo do miocárdio e morte vascular em todos os grupos etários e em ambos os sexos. ${ }^{5} \mathrm{~A}$ aspirina em doses diárias de 81 a 325 mg é eficaz na prevenção do AVC isquémico em doentes de alto risco. ${ }^{5}$ Foi recentemente demonstrado que a associação com o dipiridamol é mais eficaz que a monoterapia com aspirina na prevenção da isquémia recorrente em doentes com doença cerebrovascular. ${ }^{10}$

O uso da ticlopidina tem diminuído devido aos seus efeitos secundários.

Em geral, em estudos comparativos, o clopidogrel foi superior à aspirina na redução de eventos vasculares (AVC, EAM, isquémia crítica), tendo a maior diferença sido encontrada na doença arterial periférica dos membros inferiores. ${ }^{8}$ Apesar do clopidogrel diminuir o risco de eventos isquémicos em doentes com doença cerebrovascular sintomática, a sua superioridade em relação à aspirina não foi estatisticamente significativa. Ainda são necessários mais estudos sobre as vantagens da associação da aspirina com clopidogrel.

As guidelines actuais não recomendam um antiagregante em relação a outro e a sua selecção deve ser feita caso a caso. ${ }^{8} \mathrm{O}$ procedimento habitual, na prática, consiste na prescrição inicial de clopidogrel em doentes com doença arterial periférica concomitante e aspirina nos restantes.

\section{Terapêutica cirúrgica}

A endarterectomia carotídea (EAC) reduz o risco de recorrência de eventos vasculares cerebrais em doentes com estenose carotídea sintomática superior a $70 \%$, como foi demonstrado na década de 1990 pelos estu- dos NASCET e ECST (European Carotid Surgery Trial). O Asymptomatic Carotid Atherosclerosis Study (ACAS) demonstrou um benefício significativo da endarterectomia e tratamento médico, em relação a tratamento médico isolado, para doentes com estenoses assintomáticas de 60 a $99 \%$. Estes achados foram confirmados pelo Asymptomatic Carotid Surgery Trial (ACST), na Europa. Sempre que a doença carotídea seja considerada relevante na etiologia do AVC, a EAC deve ser realizada rapidamente, idealmente nas duas primeiras semanas após a ocorrência do AVC. ${ }^{9,10,11}$

Idosos com idade superior a 75 anos, sem disfunção cardíaca severa ou outras patologias orgânicas (como insuficiência renal crónica, doença pulmonar obstrutiva ou insuficiência hepática), caso tenham indicação cirúrgica, têm melhores resultados com a EAC, do que com o stenting carotídeo. ${ }^{12}$

No caso do sexo feminino, na estenose sintomática superior a 70\%, deve efectuar-se EAC; pelo contrário, as estenoses moderadas devem ser tratadas medicamente, ${ }^{12}$ devido ao facto de existir um menor risco de AVC no sexo feminino, quando tratado medicamente, em relação ao sexo masculino. ${ }^{12}$ Existe, também, evidência de que o risco de AVC peri-operatório é superior no sexo feminino, quando submetido a EAC. ${ }^{11}$

A EAC pode estar indicada em alguns doentes com estenose de 50-69\% sem défice neurológico grave. Isto é apenas válido para centros com uma taxa de complicações peri-operatórias (AVC/morte) inferior a $3 \%{ }^{3} \mathrm{O}$ subgrupo de doentes que mais parecem beneficiar da cirurgia é o dos homens com sintomas hemisféricos recentes.

Existe evidência para a realização de EAC nas situações anteriormente descritas, mas não para o stenting carotídeo. Este último deve ser recomendado para os doentes com irradiação prévia do pescoço, cirurgia radical do pescoço ou traqueostomia, por se tratar de um pescoço hostil. Deve ser preferido em doentes com estenoses recorrentes, pescoço imobilizado, uma bifurcação carotídea alta ou oclusão contralateral, mas, também, quando existe um risco cirúrgico muito elevado, como na doença pulmonar ou cardíaca severa. ${ }^{3,8,9,12}$

A EAC não está recomendada em doentes com estenose inferior a $50 \%$ ou caso haja oclusão completa, em que apenas está recomendado o tratamento médico. $^{3,8,9,12}$ 


\section{CRITÉRIOS DE REFERENCIAÇÃO}

- Estenose carotídea sintomática superior a $70 \%$

- Estenose carotídea sintomática de 50-69\%, sem défice neurológico grave, sobretudo se doente do sexo masculino com sintomas hemisféricos recentes.

- Estenose carotídea assintomática de 60 a 99\% Caso não preencha os critérios de referenciação, deverá realizar ecodoppler anual e os exames laboratoriais anteriormente referidos.

Os aspectos mais importantes encontram-se resumidos na Figura 1.

\section{CONCLUSÕES}

Devido à importante morbilidade, incapacidade e mortalidade que se lhe associam, a doença aterosclerótica carotídea deve ser alvo da maior atenção.

A sua prevenção e correcto tratamento poderão levar à diminuição da sua complicação mais grave, o AVC.

Assim, o Médico de Família deverá, na sua prática clínica, identificar os doentes com maior risco para este problema e promover a correcção dos factores que se lhe associam. O diagnóstico precoce, o tratamento médico e o conhecimento das indicações para terapêuti-

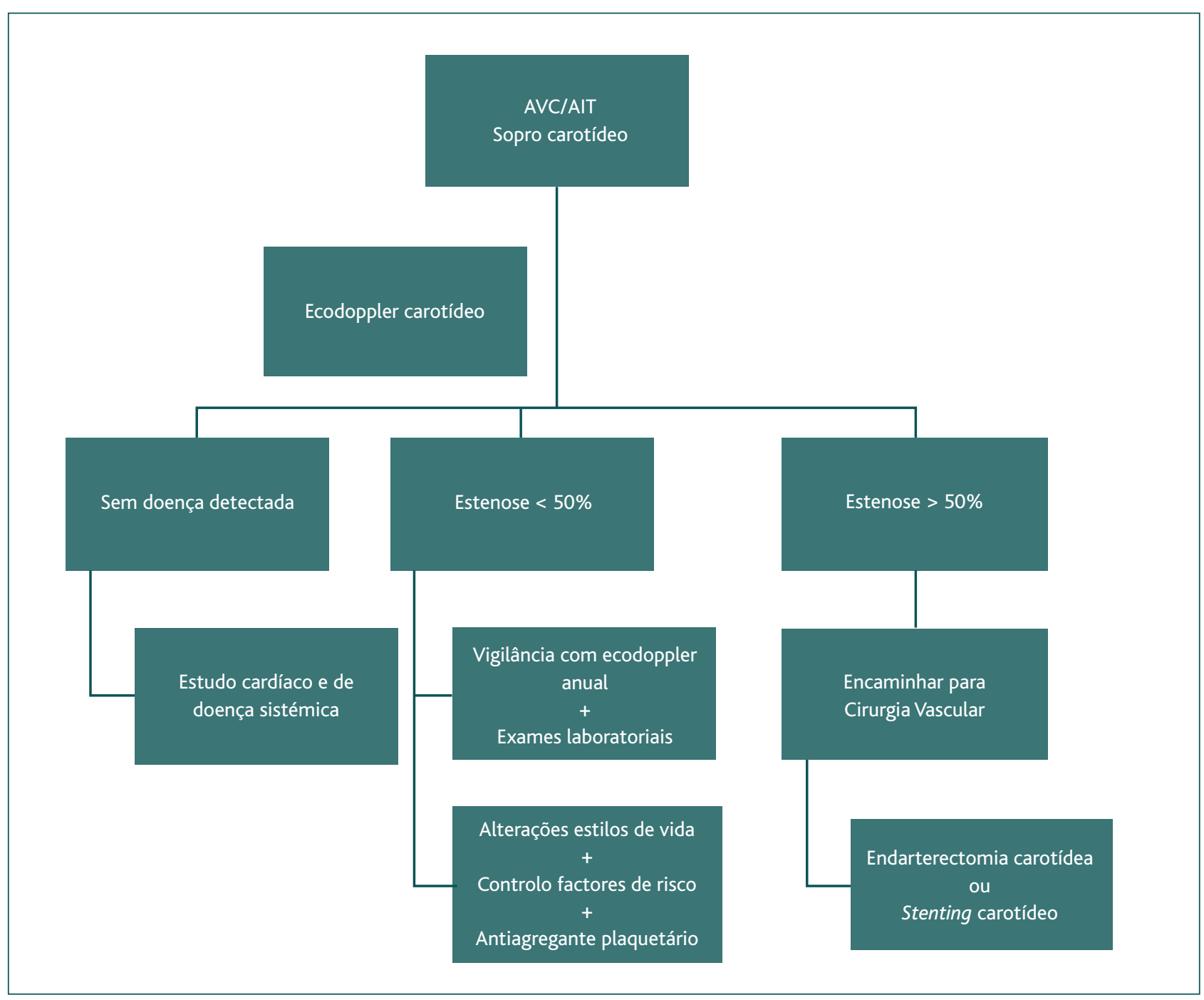

Figura 1. Resumo do tratamento, follow up e critérios de referenciação. 
ca cirúrgica, com uma referenciação adequada a Cirurgia Vascular são os outros aspectos importantes em que pode intervir.

\section{REFERÊNCIAS BIBLIORÁFICAS}

1. O'Brien JT, Erkinjuntti T, Reisberg B, Roman G, Sawada T, Pantoni L, Bowler JV, Ballard C, DeCarli C, Gorelick PB, Rockwood K, Burns A, Gauthier S, DeKosky ST:Vascular cognitive impairment. Lancet Neurol 2003;2:89-98

2. Centers for Disease Control and Prevention (CDC). Stroke facts and statistics. 2/9/2007.

3. William C. Makey. Cerebrovascular Disease: General Considerations; Timothy F. Kresowik and Harold P. Adams, Jr. Cerebrovascular Disease: Decision making and medical treatment. In Rutherford s Vascular Surgery 7th Edition. 2010.

4. Wardlaw JM, Chappell FM, Stevenson M, et al. Accurate, practical and costeffective assessment of carotid stenosis in the UK. Health Technology Assessment. 2006; 10.

5. U.S. Preventive Services Task Force. Screening for Carotid Artery Stenosis: U.S. Preventive Services Task Force Recommendation Statement. Annals of Internal Medicine. December 18, 2007 vol. 147 no. 12 854-859.

6. Lopez AD, Mathers CD, Ezzati M, Jamison DT, Murray CJ: Global and regional burden of disease and risk factors, 2001: systematic analysis of population health data. Lancet 2006;367:1747-1757

7. Reynolds K, Lewis B, Nolen JD, Kinney GL, Sathya B, He J: Alcohol consumption and risk of stroke: a meta-analysis. JAMA 2003;289:579-588.

8. Sacco RL, Adams R, Albers G, et al. Guidelines for prevention of stroke in patients with ischemic stroke or transient ischemic attack: a statement for healthcare professionals from the American Heart Association/American Stroke Association Council on Stroke: Co-sponsored by the Council on Cardiovascular Radiology and Intervention. Stroke. 2006;37:577-617.

9. Mendes Pedro L. Timing da intervenção carotídea em lesões sintomáticas. Angiologia e Cirurgia Vascular. Volume 6, Número 1, 7-10, Março 2010.

10. CAPRIE Steering Committee. A randomised, blinded, trial of clopidogrel versus aspirin in patients at risk of ischaemic events (CAPRIE). The Lancet, Volume 348, Pages 1329 - 1339, 16 November 1996.

11. Rothwell P, Eliasziw M, Gutnikov S, Warlow C, HJM B, for the Carotid Endarterectomy Trialists Collaboration: Endarterectomy for symptomatic carotid stenosis in relation to clinical subgroups and timing of surgery. Lancet 2004;363:915-924.

12. Chambers BR, Donnan GA: Carotid endarterectomy for asymptoma-tic carotid stenosis. Cochrane Database Syst Rev 2005:CD001923

\section{CONFLITOS DE INTERESSE}

Os autores declaram não exisitir conflitos de interesse na elaboração deste artigo

\section{ENDEREÇO PARA CORRESPONDÊNCIA}

Inês Müller Pinheiro

Serviço de Cirurgia Vascular, Hospital Garcia de Orta, E.P.E.

Av. Prof. Torrado da Silva

2801-951 Almada - Portugal

E-mail: inesmullerpinheiro@gmail.com

Recebido em 30/08/2010

Aceite para publicação em 18/10/2010

\section{ABSTRACT}

\section{CAROTID ARTERY DISEASE}

About $90 \%$ of the cases of extracranial cerebrovascular disease are due to atherosclerosis. The atherosclerosis plaques occur mainly in the carotid bifurcation and their most dreaded consequence is a stroke, which is the number one cause of morbilitity and permanent disability in Europe.

It is also considered the second most important cause of dementia, the most important cause of epilepsy in the elderly and a frequent cause of depression.

Roughly $80 \%$ of the strokes are ischemic, $20 \%$ of which are due to extracranial carotid atherosclerosis releasing emboli into the cerebral circulation or due to insufficient flow. The carotid duplex scan is the first line diagnostic method. The objective of all therapeutic intervention is to prevent cerebral infarction.

Current stroke prevention guidelines recommend dietary, lifestyle, and risk factor modification. Antiplatelet therapy is currently used to prevent thromboembolic complications of carotid disease. Patients with a $70 \%$ or greater symptomatic carotid stenosis have clear indications for surgical intervention (endarterectomy or stenting).

Sixty to $99 \%$ asymptomatic carotid stenosis should only be operated in high risk patients and carotid stenting should be avoided.

Keywords: Stroke; Atherosclerosis; Carotid Artery Disease; Platelet Inhibitors. 